\title{
Multi-component reactions and related chemistries
}

\author{
Maxim A. Mironov
}

Published online: 23 July 2010

(c) Springer Science+Business Media B.V. 2010

Multi-component reactions (MCRs) is one of the oldest fields in organic chemistry, which has rapidly grown during the past two decades after a relative inactive period. The first synthesis of organic molecules via MCR was published in 1850 by Strecker. Then in 1917 the first example of the use of MCR for natural product synthesis appeared with the synthesis of the alkaloid tropinone via Robinson's synthesis. The most active period in the development of the classical MCR chemistry was in the 1960s when the research groups of Ivar Ugi and Ulrich Schoellkopf (Germany) and Albert van Leusen (Netherlands) discovered isocyanide-based MCRs. Now these reactions have become powerful tools for the synthesis of peptides, pyrroles, imidazoles and indoles. However, for quite sometime MCR chemistry was considered exotic in organic synthesis and its development plateaued.

The situation changed with development of parallel synthesis, combinatorial chemistry and high-throughput experimentation. The number of research groups active in the MCR field grew rapidly in the past 10 years, and novel MCRs are being discovered just about every week resulting in several hundreds publication per year. Now more and more companies include MCR chemistry in their synthetic arsenal because (1) these chemistries are fully amenable to automation since there is no need to isolate intermediates and the reactions can be carried out in single reaction vessels, and (2) MCRs are more convergent based on a sequence of uniand bimolecular reactions. In addition, MCRs are a particular case of reaction networks, which can be considered as a model of natural processes and prebiotic evolution, and new possibilities for MCR development appeared with the

M. A. Mironov ( $\varangle)$

Ural Federal University, Ekaterinburg, Russian Federation

e-mail:mirma@mail.ustu.ru; mirma@e1.ru introduction of Diversity-Oriented Synthesis (DOS). Creating and introducing structural diversity and complexity cores followed by post-transformations offer an efficient synthesis platform to produce novel and attractive compound collections.

The new progress and interest in MCRs led to the birth of MCR-focussed conferences. In October 2000 the "First International Conference on Multi Component Reactions, Combinatorial and Related Chemistry" was held in Munich, Germany, with Ivar Ugi as chairman, who can be considered the father of the concept of MCRs and the grandfather of combinatorial chemistry. This event was the starting point of MCR conference series: MCR2003 in Genova, Italy, and MCR2006 at the Royal Tropical Museum in Amsterdam, Netherlands. These conferences were highly successful with ca. 250 participants, about half of them coming from private companies.

The most recent conference took place in Ekaterinburg, Russia, in May 2009: MCR2009. This conference was chaired by Prof. Oleg Chupakhin and me, where scientists from 17 countries covering Europe, North America, South Asia and the Pacific region attended and discussed recent progress and development in the field.

It is my pleasure to present you this special issue of Molecular Diversity dedicated to the MCR2009 conference. This is a collection of major contributions from established scientists covering a broad scope of MCR topics currently discussed in the MCR community. The role of MCRs in the pharmaceutical industry is also discussed, and you can find here articles and mini-reviews devoted to the applications of MCRs for drug design.

I hope that you enjoy it. 